\title{
SUS, MODELOS ASSISTENCIAIS E VIGILÂNCIA DA SAÚDE*
}

\author{
Carmem Fontes Teixeiral', Jairnilson Silva Paim², Ana Luiza Vilasbôas ${ }^{3}$
}

\begin{abstract}
Resumo
O processo de construção do Sistema Único de Saúde no Brasil tem contemplado a implementação de um conjunto de estratégias de mudança do financiamento, gestão e organização da produção de serviços. Nesse contexto ganha importáncia o debate sobre a municipalização da gestão do sistema e as alternativas da redefinição do(s) modelo(s) assistencial(ais) do SUS. Este artigo apresenta uma sistematização teóricoconceitual e metodológica sobre a Vigilancia da Saúde, entendida como um enfoque que pode contribuir para a atualização das concepçoes que orientam a reorganização das práticas de savide ao nivel municipal e revisam os principais métodos e técnicas que podem ser utilizados nesse processo. Enfatiza o uso da epidemiologia e das ciências sociais em saúde na análise da situação de saúde da população, no planejamento e programação local e na organização de operaçôes dirigidas ao enfrentamento de problemas especificos, em territórios delimitados, com ênfase nas açôes intersetoriais e setoriais de promoção da saúde, prevenção de riscos e agravos, e reorganização da assistência médico-ambulatorial e hospitalar.
\end{abstract}

Palavras-Chave: Vigilancia da Saúde; Modelos Assistenciais; Epidemiologia em Serviços de Saúde; Planejamento e Programação Local em Sauide; Processo de Trabalho em Saúde.

\section{Summary}

The organizational process of the Brazilian National Health System has implemented strategic changes in its financing and management as well as in the health care services. In this context, the debate on the municipalization of the system management and on alternative assistance models is extremely important. The objective of this article is to present a theoretical and methodological systematization of the health surveillance concept, in order to contribute to the reorganization process in health practice at the municipal level. The text emphasizes the use of epidemiology and social sciences in the analysis of the population's bealth situation and in the planning and organization of activities to confront specific problems in defined areas. Emphasis is given to intersectorial and sectorial actions in health promotion, disease prevention, and medical assistance at ambulatory and hospital levels.

Key-Words: Health Surveillance; Assistance Models; Epidemiology the Health Service; Local Health Planning; Health Pratices.

\footnotetext{
* Texto elaborado para a Oficina de Vigilancia em Saúde do IV Congresso Brasileiro de Epidemiologia.

${ }^{1}$ Professora do Instituto de Savide Coletiva da UFBa.

${ }^{2}$ Professor do Instituto de Saúde Coletiva da UFBa.

${ }^{3}$ Mestranda em Saúde Comunitária - ISC/UFBa.

Endereço para correspondência: Instituto de Saúde Coletiva. Universidade Federal da Babia. Rua Padre Feijó, 29. Salvador/BA. CEP: 40.110-170
} 


\section{Introdução}

O processo de construção do Sistema Único de Sande (SUS) vem sendo marcado pela elaboração e implementação de instrumentos legais e normativos, cujo propósito central é a racionalização das formas de financiamento $e$ gestão dos sistemas estaduais e municipais de saude, fundamentados em uma proposta de ampliação da autonomia politica dos municipios, enquanto base da estrutura politicoadministrativa do Estado.

Nesse contexto, o debate politicoinstitucional tem privilegiado os "componentes" financiamento e gestão do SUS. ${ }^{1}$ Também tem sido discutida, a partir do processo de descentralização, a questão da "organização do sistema", especialmente no que diz respeito à redefinição de funções e competências do Ministério da Saúde (MS), das Secretarias Estaduais de Saúde (SES) e das Secretarias Municipais de Saúde (SMS), à reestruturação da Fundação Nacional de Saúde (FNS) e à redefinição das relações com o setor privado, esta última em função das propostas do Ministério da Administração e Reforma do Estado, sugerindo a criação das chamadas "organizações sociais". ${ }^{2}$

$O$ debate das macropoliticas no setor sauide, portanto, não tem privilegiado a questão dos modelos assistenciais, isto é, das formas de organização tecnológica do processo de prestação de serviços de saúde. O sistema de sanide brasileiro é hoje, assim, palco da disputa entre modelos assistenciais diversos, com a tendência de reprodução conflitiva dos modelos hegemônicos, ou seja, o modelo médicoassistencial privatista (ênfase na assistência médico-hospitalar e nos serviços de apoio diagnóstico e terapêutico) e o modelo assistencial sanitarista (campanhas, programas especiais e ações de vigilância epidemiológica e sanitária), ao lado dos esforços de construção de "modelos" alternativos. ${ }^{3,4}$

$$
\text { Esse processo tem contemplado }
$$

tentativas de articular ações de promoção, prevenção, recuperação e reabilitação, em uma dupla dimensão, individual e coletiva, que passaram a ser operacionalizadas no processo de distritalização dos serviços de saúde desencadeado a partir do Sistema Único e Descentralizado de Saúde - SUDS (87-89) e desenvolvido, posteriormente, em alguns municipios do pais. ${ }^{3,5,6}$ Desse modo, ao nivel micro, vem se acumulando experiência na construção de "modelos alternativos" ao modelo assistencial hegemônico, incorporando, de certa forma, métodos, técnicas e instrumentos provindos da epidemiologia, do planejamento $e$ das ciências sociais em sande. Estas experiências apontam possibilidades concretas de construção de um "modelo de atenção à saúde voltado para a qualidade de vida", ${ }^{7}$ tal como proposto no temário da $10^{a}$ Conferência Nacional de Saúde. ${ }^{8}$

Essas possibilidades foram reconbecidas no Encontro de Secretários Municipais de Sauide realizado no Ceará, em 1995, no qual foi elaborada a "Carta de Fortaleza"," documento que reconbece as conferências de Alma-Ata, em 1978, Ottawa, em 1986, e Bogotá, em 1992 como "marcos referenciais do conceito de sanide para todos como direito fundamental do ser humano". Ao considerar, também, as experiências em curso, explicitou a seguinte posição:

"A crise do financiamento do modelo de sauide centrado na doenca exige o estabelecimento de novas estratégias que recuperem o paradigma da saúde centrado na qualidade de vida e desenvolvimento global das comunidades com participação dos cidadãos. (...) é possivel vishumbrar metas comuns que valorizem a importancia das açôes intersetoriais e de promoção da sauide ao mesmo tempo que seguir buscando formas autónomas e criativas para a atenção integral à sauide. (...) Oexemplo brasileiro neste campo demonstra que é possivel a construcão de um novo paradigma em sanide em nivel municipal a partir de um processo integrado, participativo e criativo que dependa fundamentalmente da decisão politica das autoridades locais." 
Para além do intercâmbio de experiências $e$ da elaboração de princípios e diretrizes gerais que norteiem as diversas iniciativas desencadeadas nos municipios, consideramos necessária a sistematização de elementos conceituais, metodológicos e instrumentais que contribuam para a adoção de decisões $e$ implementação de ações no âmbito municipal, tendo como propósito a construção do(s) modelo(s) assistencial (ais) coerentes com a problemática de cada municipio e viáveis do ponto de vista da disponibilidade de recursos e da capacidade técnica, gerencial e politica dos sistemas municipais de saúde.

Nessa perspectiva é que nos propomos, no presente texto, a apresentar uma sistematização preliminar, com o objetivo central de contribuir para o debate que se trava hoje em torno da Vigilância da Saúde, entendida como eixo de um processo de reorientação do(s) modelo(s) assistencial (ais) do SUS. Para isso, procuramos discutir o significado da Vigilancia da Saúde no contexto da municipalização para, em seguida, revisar o debate conceitual sobre Vigilância da Saúde. Por último, sistematizamos algumas propostas para a operacionalização das práticas de Vigilancia da Saúde no ámbito municipal.

\section{A Vigilância da Saúde no contexto da municipalização}

$O$ processo de municipalização, na medida em que venha a significar uma efetiva redefinição de funções e competências entre os niveis de governo do SUS, implica a constituição de "sistemas municipais de saúde", nos quais se pode identificar o modelo de gestão $e$ de atenção à saúde ou "modelo assistencial". ${ }^{10}$ Antes do SUS e especificamente antes da implementação da NOB 001/93, não se poderia considerar que os municipios brasileiros tivessem "sistemas municipais". Os municipios tinham serviços de saúde municipais, porém não tinham capacidade de gestão do conjunto das instituiçôes e unidades de prestação de serviços de saúde localizadas em seus territórios.
Embora a preocupação central naquele momento fosse com a descentralização da gestão da rede de serviços de prestação direta a pessoas (assistência médico-ambulatorial), na forma de "gestão parcial", buscava-se induzir o municipio a assumir as açôes de vigilância epidemiológica e sanitária, predominantemente sob a órbita das Secretarias Estaduais de Saúde e, em várias regiões e microrregiões, sob controle da Fundação Nacional de Saúde. Somente quando atingia o estágio de "gestão semiplena" é que o município passava a atuar como gestor do sistema como um todo, assumindo a responsabilidade também sobre a atenção hospitalar, de maior complexidade e maior custo.

A implementação da NOB 001/93, ${ }^{11}$ além de não ter sido completada em todos os municipios do pais, resultou em uma relativa reconcentração de recursos financeiros em regiões, estados e municipios, em função, principalmente, da manutenção dos critérios de repasse de recursos financeiros, que se baseavam fundamentalmente na capacidade de produção de serviços. Ora, por esta lógica, os municipios dotados de maior infra-estrutura e capacidade gerencial passaram a disputar uma parcela mais significativa dos recursos federais para a saúde. Em um contexto no qual estes recursos foram reduzidos, o conflito redistributivo acirrou-se, passando a constituir o tema central da agenda politica.

Paralelamente a este debate em torno do financiamento e gestão do SUS, o Ministério da Saúde desenvolveu a "estratégia" de Saúde da Familia, ${ }^{12}$ cujos resultados positivos em termos do impacto sobre alguns indicadores de saúde vêm contribuindo para legitimá-la, a ponto de ser considerada hoje o eixo do processo de reorganização dos serviços básicos no SUS. Do mesmo modo, esforços mais recentes no ámbito do Centro Nacional de Epidemiologia CENEPI, acenam com a possibilidade de apoio financeiro e técnico para a implementação de sistemas de "vigilância da saúde", aí entendidos como vigilância epidemiológica, sanitária e 
ambiental, em um amplo programa denominado VIGISUS. ${ }^{13}$

Durante a elaboração da NOB 001 $/ 96^{14}$, além da tentativa de definir um critério populacional padrão para a definição do volume de recursos financeiros que caberia a cada municipio habilitado para as ações básicas, foram introduzidos diversos "fatores de estímulo" à implementação de inovações, entre as quais o Programa de Saúde da Família (PSF) e as ações de vigilância epidemiológica e sanitária.

O que importa ressaltar é que, nesse contexto, o municipio tem condições de articular o conjunto das propostas, programas e estratégias que vêm sendo definidas no nivel federal e em vários estados para desencadear, em seu ámbito, um processo de reorientação do "modelo assistencial” do SUS que não signifique a mera reprodução do "modelo médico-assistencial privatista", subordinando o "modelo sanitarista", ou seja, a chamada "inampização"do SUS. ${ }^{15}$
Pelo contrário, levando em conta a existência de instrumentos financeiros como o Piso Assistencial Básico (PAB fixo e variável), gerenciais e técnico-operacionais a exemplo da Programação Pactuada Integrada (PPI), do Programa de Agentes Comunitários de Saúde (PACS), do PSF e do VIGISUS, que podem ser utilizados para a criação de uma proposta que aponta em outra direção, o município pode caminhar para a construção de um modelo fundamentado na Vigilância da Saúde.

A Figura 1 sintetiza essa possibilidade de atuação do município, articulando, para cada um dos niveis de atenção, as distintas propostas $e$ "projetos estruturantes" que se encontram em debate na presente conjuntura. ${ }^{16}$ Neste esquema, porém, o distrito sanitário abrange os três niveis de atenção e o PSF não está confinado na Atenção Primária à Saúde (APS). A saúde da familia "invade" os niveis de atenção secundária e terciária na medida em que sua equipe,

Figura 1 - Vigilância da Saúde

\section{VIGILÂNCIA DA SAÚDE}

POLÍTICAS PÚBLICAS PROMOÇÃO À SAÚDE

ATENÇÃO PRIMÁRIA

\author{
ATENÇÃO \\ SECUNDÁRIA \\ E TERCIÁRIA
}

SAÚDE DA FAMÍLIA

(PACS / PSF)

CIDADE SAUDÁVEL 
particularmente o médico e a enfermeira, pode se responsabilizar pelo paciente e pelo apoio à sua familia, acompanbando-o na atenção especializada, inclusive na assistência hospitalar. Haveria situações em que o médico de familia, respeitados os preceitos éticos em relação aos seus colegas do hospital, discutivia procedimentos diagnósticos e terapêuticos, além de proceder visitas hospitalares durante a internação do seu paciente.

Vigilância $e m$ saúde e Vigilância da Saúde

\section{A vigilância no campo da Saúde Pública}

Em um trabalho elaborado na segunda metade dos anos 70, Juan César Garcia chamava a atenção para a evolução dos enfoques que se desenvolveram ao longo da história da Saúde Pública, comentando sua similitude com a evolução da "arte da guerra” que partia da idéia inicial de "eliminação do inimigo", oriunda da "guerra de movimento", traduzida no campo da Saúde Pública com a noção de "erradicação", passando pela idéia de "controle", provinda da "guerra de posição", até a noção de "vigilância" que corresponderia ao periodo da "guerra fria". ${ }^{17}$

De fato, o desenvolvimento conceitual, metodológico e instrumental da Saúde Pública contemporánea corresponde aos avanços da bacteriologia e parasitologia, a partir do final do Século XIX, com as descobertas que possibilitaram o estabelecimento de estratégias de combate às doenças infeciosas e parasitárias, através, basicamente, de práticas que, tomando como objeto os “modos de transmissão", 18 utilizam técnicas de controle de vetores, saneamento ambiental e educação sanitária das populações.

Do ponto de vista operacional, essas estratégias apresentaram uma mutação em suas finalidades, dos objetivos iniciais de "erradicação" de determinadas doenças, como aliás veio a acontecer com a variola $e$ presentemente com a poliomielite, para a constatação das dificuldades de erradicação de algumas doenças, base das propostas de "controle", originadas do combate à malária e à tuberculose em meados do século $X X$, até a assimilação da noção de "vigilância epidemiológica", que passou a ser usada mais amplamente a partir dos anos $50 .^{19}$

$O$ desenvolvimento da Epidemiologia, já nas primeiras décadas deste século, registra tentativas de expansão do seu objeto para além das doenças infecto-contagiosas, desenvolvendose, nas décadas de 30 e 40, esforços de sistematização teórica do conceito de "risco". Só a partir dos anos 60, porém, com o extraordinário desenvolvimento das técnicas de computação de dados, é que esta disciplina adquire a autonomia que a caracteriza atualmente como eixo da produção de conbecimentos sobre problemas de saúde em uma perspectiva coletiva. ${ }^{20}$

$A$ incorporação da noção de "risco" $e$ especialmente a busca de identificação dos "fatores de risco" envolvidos na determinação das doenças, não só as infecto-contagiosas mas principalmente as crônico-degenerativas, que passavam a ocupar um lugar predominante no perfil epidemiológico das populações em sociedades industriais, ${ }^{21}$ vem provocando a modernização das estratégias de ação no campo da Saúde Pública, tanto pela ampliação $e$ diversificação do seu objeto quanto pela incorporação de novas técnicas e instrumentos de geração de informações e organização das intervenções sobre "danos", "indícios de danos", "riscos" e "condicionantes e determinantes" dos problemas de saúde. ${ }^{22}$

Desse modo, além da ampliação do objeto dos "programas de controle" que tendem a ultrapassar o limite estreito das doenças infecciosas e parasitárias, dirigindo-se a grupos populacionais expostos a riscos diferenciados de adoecer e morrer, a exemplo dos programas de "saude materno-infantil", "saude do trabalbador", "saude do idoso", etc., vêm se observando, notadamente a partir dos anos 70, a formulação e implementação de propostas 
dirigidas à montagem de "sistemas de vigilância epidemiológica", cuja tradução operacional pretende ser uma ampla rede de unidades geradoras de dados que permitam a adoção de decisões e a execução de ações de investigação e controle.

$O$ desenvolvimento institucional da Saúde Pública no Brasil, ilustra, com algumas particularidades, a evolução conceitual e a modernização tecnológica e operacional que apontamos acima. Das campanhas sanitárias do inicio do século (sanitarismo campanhista), aos sistemas de vigilância epidemiológica propostos em meados dos anos 70, no contexto das politicas racionalizadoras desencadeadas com os chamados Programas de Extensão de CoberturaPECS, até o debate sobre a integração" entre a vigilância epidemiológica e a vigilância sanitária na segunda metade dos anos 80, na época da implantação do SUDS.

De fato, a institucionalização dos programas de erradicação e controle e a implantação da vigilância no Brasil, ao longo dos viltimos 90 anos, implicou, do ponto de vista político-institucional, a organização centralizada (federal) de órgãos e departamentos responsáveis pelas campanhas e programas, ao tempo em que se cristalizava uma distinção entre a vigiláncia epidemiológica, voltada para o controle de “casos" e "contatos", e a vigilância sanitária, voltada para o controle de "ambientes, produtos e serviços”.

Embora se possa considerar que do ponto de vista técnico-operacional há especificidades que justificam a existência dessas "vigilâncias", a primeira, a epidemiológica, obedecendo a uma racionalidade técnicosanitária fundada na clínica e na epidemiologia, e a segunda, obedecendo a uma racionalidade politico-jurídica, fundada nas normas que regulamentam a produção, distribuiçãa $e$ consumo de bens e serviços, ${ }^{23}$ não se justificaria a sua institucionalização como órgãos separados, particularmente no ámbito municipal.

Em meados dos anos 70, quando se difundiu a concepção sistêmica, foi proposta a criação do SNVE - Sistema Nacional de Vigilância Epidemiológica, estabelecendo-se as bases legais (Lei $n^{\circ}$ 6.259) e promovendo-se uma certa desconcentração das açôes para as Secretarias de Sanide dos estados. ${ }^{24}$ Nos anos 80, especialmente com o SUDS, dinamizou-se $o$ debate sobre a ampliação do objeto da vigilancia epidemiológica, ${ }^{25}$ ao tempo em que se desencadeou uma reflexão sobre a vigilância sanitária, a partir da constatação da fragilidade dos órgãos nacional e estaduais responsáveis por estas práticas. Isto gerou, inclusive, a reflexão sobre os limites e possibilidades de "integração" institucional das "vigilâncias", debate ainda atual, na medida em que se avance para a formulação e implementação de um sistema de Vigilância da Saúde como proposto, inclusive, em uma oficina de trabalho realizado no Congresso Brasileiro de Epidemiologia realizado $\mathrm{em} 1995 .^{26}$

A fundamentação dessa proposta se baseia, de um lado, nos avanços conceituais, metodológicos e instrumentais no campo da Epidemiologia Crítica $^{18}$ e, de outro, na análise do contexto politico-institucional decorrente do processo de construção do SUS, especialmente no que se refere à descentralização das açôes de vigilância da sauide para os municípios. Para discutir esse processo, na perspectiva do municipio, é importante revisar, brevemente, o debate atual na área, identificando as vertentes conceituais $e$ as propostas metodológicas existentes.

\section{O debate sobre Vigilância da Saúde na América Latina}

As distintas vertentes do debate em torno da Vigilancia se expressam na utilização de variaçôes terminológicas como "Vigilância da Saúde", "Vigilância à Saúde" e "Vigilância em Sauide". O eixo comum é a abertura para a epidemiologia, ${ }^{27}$ tanto no que diz respeito à sua contribuição para a análise dos problemas de saúde que transcenda a mera sistematização de indicadores gerais, quanto no ámbito do 
debate sobre planejamento e organização de sistemas e serviços, ${ }^{28}$ isto é, na implantação de novas práticas e novos "modelos assistenciais".

As tentativas de aproximação entre a epidemiologia, o planejamento e a organização dos serviços, enquanto um "movimento", organizado institucionalmente que transcende o interesse e a iniciativa singular de um ou outro pesquisador, dirigente ou técnico, ganharam força nos anos 80, a partir dos eventos realizados após o Seminário sobre "Usos e perspectivas da Epidemiologia", ${ }^{29}$ realizado em Buenos Aires. As duas temáticas, porém, ainda apareciam separadas: de um lado, a planificação e programação de sistemas de serviços; de outro, a elaboração de programas dirigidos ao controle de doenças especificas, ou programas dirigidos a grupos populacionais especificos, nos quais se incluiam ações de promoção da saúde, prevenção de agravos e recuperação.

Já na Conferência da ALAESP Associação Latinoamericana e do Caribe de Educação em Saúde Pública, realizada no México em 1987, aparece explicitada uma análise crítica dos processos desencadeados a partir do Seminário de 1983 , identificando-se limitações no exercício da capacidade analítica da situação de saúde prevalente nos paises da região, apontando-se a necessidade de que "a investigação e análise sistemática da situação de sanide deve reforcar nos paises a capacidade para um maior e melhor uso dos dados de mortalidade e morbidade na identificação de prioridades e na avaliação dos resultados das açôes dos programas e serviços" 30

Um dos documentos básicos do encontro abordava especificamente a "Epidemiologia e organização de serviços" apontando o interesse renascente, sobretudo em centros de investigação e ensino, no desenvolvimento de estudos de grupos especificos de população, buscando-se evidenciar as relaçôes entre condiçôes de vida $e$ trabalho com a situação de saúde. O autor, Pedro Luis Castellanos, sugere que "para recuperar o enorme potencial que hoje encerram as técnicas quantitativas para a avaliação de riscos e associaçôes causais, a fim de se alcancar um maior impacto na planificação e gestão dos serviços de saúde, será necessário que a epidemiologia recupere, como espago privilegiado de investigação causal, a explicação da situação de sauide de grupos especificos da população $e$ avaliação de relaçôes causais mais complexas e menos lineares que as de causa-efeito" ${ }^{31}$

Castellanos desenvolve várias considerações sobre a difusão que vinha sendo feita das "estratégias de alto risco", derivadas de conbecimentos sobre os fatores de risco de casos individuais de uma enfermidade, em contraposição à chamada "estratégia populacional" ou de "saúde pública", na conformação dos modelos assistenciais dos paises latino-americanos. Ainda que reconbeça certas vantagens das estratégias de "alto risco", chama a atenção para que estas tendem a estimular o desenvolvimento de modelos assistenciais verticais ou especificos para alguma enfermidade, tais como os programas de erradicação e controle de doenças transmissíveis fomentados pelas agências internacionais desde o inicio do século. Entre suas deficiências, o autor aponta que este tipo de programa contribui muito pouco para o desenvolvimento de serviços permanentes de saúde para a população geral, ademais de ser pouco estimulante da participação popular. ${ }^{31}$

A temática que emergiu e passou a interessar crescentemente tanto planificadores quanto epidemiólogos interessados nas questões relacionadas com os serviços, na segunda metade dos anos 80, foi a dos "modelos assistenciais". No final da década de 80 e início da de 90, a OPS desencadeou uma reflexão coletiva em torno do MPPS - Modelo de Prestação de Serviços de Saúde, promovendo reuniões especificas sobre os distintos "componentes" deste modelo segundo a concepção já clássica dos niveis de prevenção oriundos da Medicina Preventiva: Promoção da Saúde (julbo de 1990), Prevenção de Enfermidades (fevereiro de 1990) $e$ Recuperação (setembro de 1989). Uma das 
conclusões mais importantes desses encontros foi a constatação de quão pouco desenvolvidos se encontravam, nos diversos paises, os componentes de Promoção (praticamente inexistente) e o de Prevenção (restrito na maioria das vezes aos programas tradicionais da Saúde Pública, inclusive as ações de Vigilância epidemiológica e sanitária). ${ }^{32}$

3. As vertentes do debate sobre Vigilância da Saúde no Brasil

No Brasil, o debate sobre a articulação entre a epidemiologia, o planejamento e a organização dos serviços foi contemplado durante o II Congresso Brasileiro de Epidemiologia realizado em Belo Horizonte, em 1992. Nesta ocasião, Guilherme Rodrigues da Silva chamou a atenção para a importância do modelo proposto por Castellanos (1992) para a análise da situação de saúde e suas tendências na população, destacando a ênfase concedida pelo autor à reprodução social das condiçôes de vida e seu reflexo como problemas de saúde. Acrescenta que "seria da maior conveniéncia a sua ampliação na análise da implementação de programas e reformas dos sistemas de servicos, numa perspectiva diferente daquela predominante nas análises da realidade nacional'. ${ }^{33}$

A possibilidade de que análises mais abrangentes da situação de saúde conduzissem a propostas de reorganização dos serviços é discutida por Paim, com base em um diagrama que ilustra o processo de transição para um novo modelo assistencial, no qual a oferta organizada de serviços viesse a suplantar as açôes dirigidas ao atendimento da chamada "demanda espontânea”, bem como as ações realizadas a partir da implantação dos chamados "programas especiais" dirigidos a grupo populacionais especificos (Figura 2). ${ }^{4} A$ "organização da oferta" ou "oferta programada" seria o espaço de articulação do enfoque epidemiológico, na medida em que a programação e execução das ações e serviços deveria partir da identificação dos problemas e necessidades da população em territórios delimitados, a exemplo do que vinha ocorrendo em vários Distritos Sanitários em processo de implantação.

A preocupação com a construção de um modelo assistencial que articulasse os conbecimentos e técnicas provindos da epidemiologia, do planejamento e das ciências sociais em saúde se expressou na utilização do termo "vigilancia à saúde", definindo-se que esta "trabalha com conceituaçáo ampla do papel da epidemiologia nos serviços de saúde, incluindo avaliação e pesquisa." (...) e, "em suas propostas de ação, deve apreender a desigualdade social $e$ portanto a distribuição desigual de agravos à saúde. Essa apreensão representa um deslocamento da sua base conceitual, do exclusivo controle e/ou erradicação dos agentes para a compreensão das relaçôes sociais que definem a desigualdade". ${ }^{34}$

Já no terceiro Congresso de Epidemiologia, aparece a distinção entre uma concepção "ampla" e outra "restrita" da

Figura 2 - Diagrama de transição para o novo modelo assistencial

Modelo anterior

ao SUDS
Novo modelo assistencial

OFERTA ORGANIZADA

\section{DEMANDA ESPONTÂNEA}

\section{PROGRAMAS ESPECIAIS}


Vigilância da Saúde. ${ }^{26}$ A concepção "restrita" entende por Vigilância à Saúde, um conjunto de açôes voltadas para o conhecimento, previsão, prevenção e enfrentamento continuado de problemas de saude, selecionados e relativos aos fatores $e$ condiçoes de risco, atuais e potenciais, e aos acidentes, incapacidades, doencas- incluindo as zoonoses, $e$ outros agravos à saúde de uma população num território determinado", significando, portanto, uma ampliação da vigilância epidemiológica, com incorporação da vigiláncia sanitária, sem, entretanto, prever a reorganização do conjunto das ações e serviços de atenção à saúde, ai incluidas a intervenção sobre determinantes sociais, de um lado, e a assistência médicohospitalar, de outro.

Por seu turno, a concepção ampliada fundamentar-se-ia no diagrama proposto por Paim (Figura 3), "resgatando o desenvolvimento conceitual e metodológico que se vem verificando a partir de uma visão ampliada de Saúde e da formulação de modelos de interpretação dos determinantes, riscos, agravos e danos, à luz da moderna Epidemiologia, articulando-os em um esquema operacional que resgata e amplia o modelo clássico da História Natural das Doencas, incorporando desde as açôes sociais organizadas pelos distintos atores até as açôes especificas de prevenção de riscos e agravos, bem como as de recuperação e reabilitaça de doentes."

Paralelamente ao debate conceitual $e$ metodológico, desenvolveu-se uma reflexão sobre a organização das ações de vigilância no ámbito do SUS, contemplando aspectos politico-institucionais e operativos. ${ }^{25,35} \mathrm{Já} \mathrm{em}$ 1990, discutia-se que "a reorganização das atividades de vigilancia epidemiológica no pais, na perspectiva de um sistema único de saúde, deve levar em consideração a redefinição das funçôes próprias de cada um dos trés niveis básicos deste sistema: 0 nivel local, abrangendo um ou mais municipios, parte de um municipio e compreendendo um conjunto de unidades prestadoras de servicos; 0 intermediário ou estadual e o nivel nacional."

Chamava-se a atenção, inclusive, para que "o papel de cada nivel poderá variar de acordo com as caracteristicas da situação epidemiológica do agravo ou da doença objeto de vigilancia e também de acordo com o grau de desenvolvimento, disponibilidade de recursos e capacidade técnicooperacional das diferentes áreas geográficas". Percebe-se, portanto, a emergência de uma concepção flexível, heterogênea, baseada na própria beterogeneidade epidemiológica $e$ sanitária existente no país. ${ }^{25}$

A proposta de descentralização das ações de vigilância, partindo da análise critica do sistema vigente, considerado "burocratizado", ganba forma na primeira metade dos anos 90, quando se chegou a formular, inclusive, uma proposta de criação de "Centros de Epidemiologia a nivel local/regional" bem como a constituição de uma "estrutura estadual de controle de vetores e açóes sobre o meio", devendo "ser buscado o objetivo de promover a municipalização das açôes, através do estimulo ao desenvolvimento, nos municipios, de capacidade técnica e operacional de controle de vetores e açôes sobre o ambiente". 35

Em 1995, a proposta de organização de um "subsistema de Vigilância da Saúde no SUS" ganha contornos mais definidos, com o debate em torno de um anteprojeto de lei que define os objetivos e atribuiçôes de cada nivel do SUS. Cabe ressaltar que a proposta suscitou questionamentos sobre "os riscos de se reduzir um rico e dinámico processo social, que envolve múltiplos atores, em contextos diferenciados, com experimentação de métodos, técnicas e instrumentos diversificados, necessariamente aberto e pouco previsinel, a um "sistema" que pode tender a se colocar como uma "camisa-de-forga". ${ }^{26}$

Pelo exposto até aqui, percebe-se que o debate atual sobre a Vigilância da Saúde apresenta algumas vertentes, que poderiam ser sintetizadas como segue:

a) Vigiláncia da Saúde equivalendo a Análise de Situações de Saúde. Ainda que ampliando e redefinindo o objeto de análise situações de saúde de grupos populacionais definidos em função de suas condições de vida, 
Figura 3 - Diagrama de Vigilância da Saúde

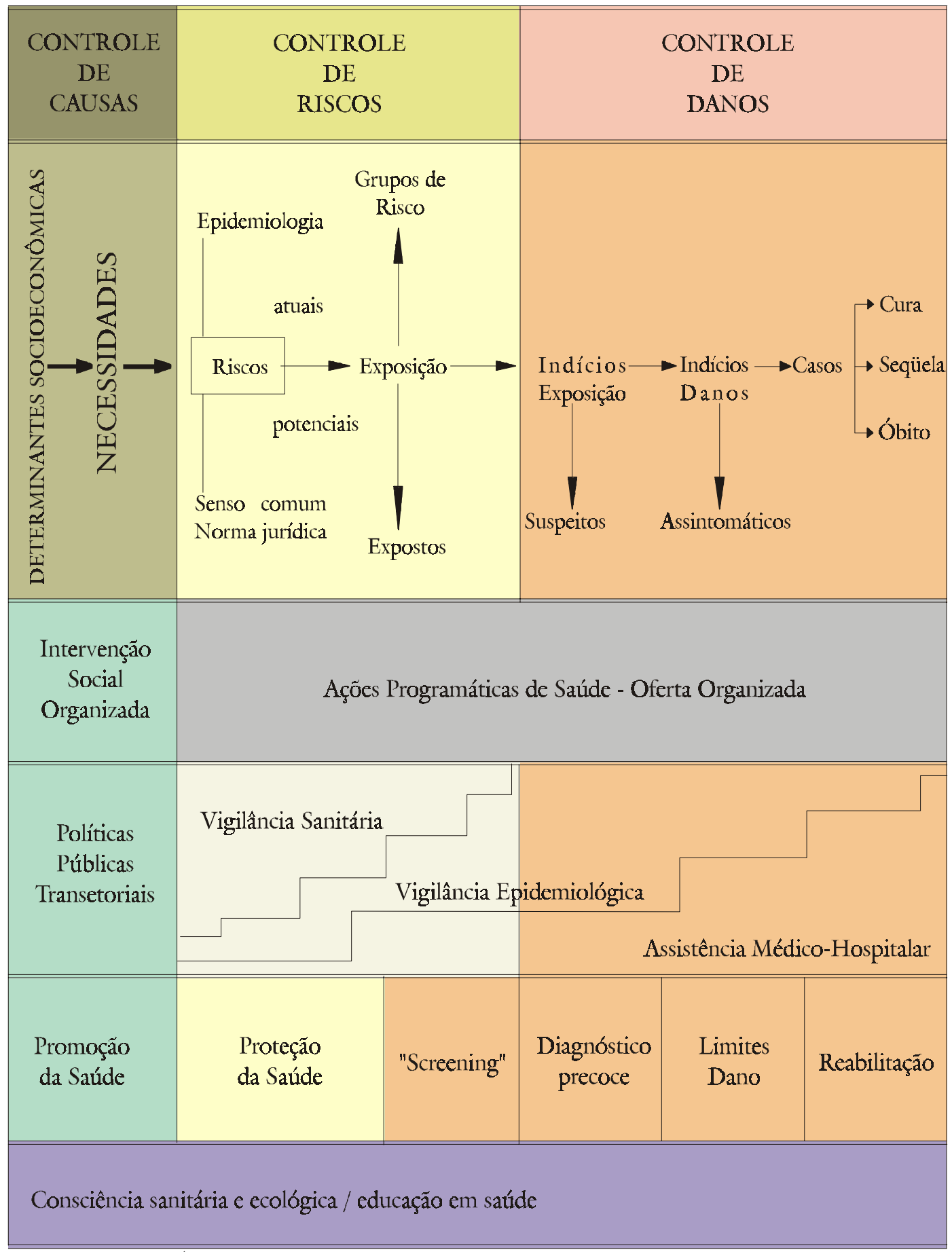

Fonte: PAIM, J.S. $1994^{4}$ 
esta acepção restringe o alcance da proposta ao monitoramento da situação de saúde, não incorporando as ações voltadas ao enfrentamento dos problemas. Do ponto de vista da prática epidemiológica em serviços tem significado uma ampliação dos objetos de vigilância epidemiológica, que passam a abarcar não apenas as doenças transmissiveis, incorporando investigaçôes e montagem de bancos de dados sobre outros agravos como mortalidade infantil, materna, doenças crônicas, acidentes e violência, como também aspectos relativos à organização e produção dos serviços de saude, contribuindo para um planejamento de saude mais abrangente. ${ }^{36,37}$

b) Vigilância da Saúde como proposta de "integração" institucional entre a Vigilância epidemiológica e a Vigiláncia sanitária, inicialmente no ámbito do processo de descentralização das ações para os órgãos estaduais (SES) e, atualmente, inserindo-se no processo de municipalização. Esta vertente se concretizou em várias das reformas administrativas levadas a cabo pelas Secretarias Estaduais de Saude na primeira metade dos anos 90, com a criação de Departamentos de Vigilância da Saúde, resultando, em alguns casos, no fortalecimento das açôes de vigiláncia sanitária e articulação com centros de saude do trabalhador, constituindo-se, entretanto, no espaço privilegiado para a implementação das campanhas de imunização e programas de controle de epidemias e endemias.

c) Vigiláncia da Saúde como uma proposta de redefinição das práticas sanitárias, havendo duas concepções, que, embora não sejam divergentes, enfatizam aspectos distintos: uma, que privilegia a dimensão técnica, ao conceber a vigiláncia à saúde enquanto um modelo assistencial alternativo conformado por um conjunto de práticas sanitárias que encerram combinações tecnológicas distintas, destinadas a controlar determinantes, riscos e danos ${ }^{4}$; outra que privilegia a dimensão gerencial da noção de vigilância à saúde, caracterizando-a como "uma prática que organiza processos de trabalho em sauide sob a forma de operaçôes, para confrontar problemas de enfrentamento continuo, num território delimitado (...) através de operações montadas sobre os problemas em seus diferentes periodos do processo saúde-doença. ${ }^{3}$

Percebe-se que a primeira definição chama a atenção para o objeto da vigilância, concebido na perspectiva das relações entre os modos de vida dos distintos grupos populacionais e as diversas expressões do processo saúde-doença. A segunda, por sua vez, destaca os meios de trabalho, isto é, os métodos, técnicas $e$ instrumentos gerenciais necessários para a operacionalização das práticas de vigilância da saude. Poderíamos acrescentar que tais práticas também diferem das ações tradicionais de vigilancia epidemiológica e sanitária ao apontarem a possibilidade de incorporação de outros sujeitos, gerentes de serviços, técnicos e representantes de grupos organizados da população.

Comparando esta concepção de Vigilancia da Saude com os modelos assistenciais vigentes (médico-assistencial e sanitarista, hegemônicos) constatam-se as diferenças com relação aos sujeitos, objeto, métodos e forma de organização dos processos de trabalbo (Figura 4). Enquanto o modelo médicoassistencial privilegia o médico, tomando como objeto a doença, em sua expressão individualizada e utiliza como meios de trabalho os conbecimentos e tecnologias que permitem o diagnóstico e a terapêutica das diversas patologias, o modelo sanitarista tem como sujeitos os sanitaristas, cujo trabalbo toma por objeto os modos de transmissão e fatores de risco das diversas doenças em uma perspectiva epidemiológica, utilizando um conjunto de meios que compóem a tecnologia sanitária (educação em saúde, saneamento, controle de vetores, imunização, etc.)

A Vigilância da Saúde, todavia, propõe a incorporação de novos sujeitos, extrapolando o conjunto de profissionais e trabalhadores de 
Figura 4 - Modelos Assistenciais e Vigilância da Saúde

\begin{tabular}{|c|c|c|c|c|}
\hline Modelo & Sujeito & Objeto & Meios de Trabalho & Formas de Organização \\
\hline $\begin{array}{l}\text { Modelo } \\
\text { médico- } \\
\text { assistencial } \\
\text { privatista }\end{array}$ & $\begin{array}{c}\text { Médico } \\
\text {. especialização } \\
\text { complementariedade } \\
\text { (paramédicos) }\end{array}$ & $\begin{array}{c}\text { Doença } \\
\text { (patologia e outras) } \\
\text { Doentes } \\
\text { (clinica e cirurgia) }\end{array}$ & $\begin{array}{l}\text { Tecnologia médica } \\
\text { (indivíduo) }\end{array}$ & $\begin{array}{c}\text { Rede de serviços de saúde } \\
\text { Hospital }\end{array}$ \\
\hline $\begin{array}{l}\text { Modelo } \\
\text { sanitarista }\end{array}$ & $\begin{array}{l}\text { Sanitarista } \\
\text { - auxiliares }\end{array}$ & $\begin{array}{c}\text { Modos de } \\
\text { transmissãa } \\
\text { Fatores de risco }\end{array}$ & Tecnologia sanitária & $\begin{array}{l}\text { Campanhas sanitárias } \\
\text { Programas especiais } \\
\text { Sistemas de vigilância } \\
\text { epidemiológica e sanitária }\end{array}$ \\
\hline $\begin{array}{l}\text { Vigilância } \\
\text { da sauide }\end{array}$ & $\begin{array}{c}\text { Equipe de sauide } \\
\text { População (cidadãos) }\end{array}$ & \begin{tabular}{|} 
Danos, riscos, \\
necessidades e \\
determinantes dos \\
modos de vida e \\
saúde (condiçôes de \\
vida e trabalho)
\end{tabular} & $\begin{array}{c}\text { Tecnologias de } \\
\text { comunicação social, de } \\
\text { planejamento e } \\
\text { programação local } \\
\text { situacional e } \\
\text { tecnologias médico- } \\
\text { sanitárias }\end{array}$ & $\begin{array}{l}\text { Politicas públicas saudáveis } \\
\text { Ações intersetoriais } \\
\text { Intervençôes especificas } \\
\text { (promoção, prevenção e } \\
\text { recuperação) } \\
\text { Operaçôes sobre problemas e } \\
\text { grupos populacionais }\end{array}$ \\
\hline
\end{tabular}

saúde ao envolver a população organizada, o que corresponde à ampliação do objeto, que abarca, além das determinações clínicoepidemiológicas no ambito individual e coletivo, as determinações sociais que afetam os distintos grupos populacionais em função de suas condiçôes de vida. Nessa perspectiva, a intervenção também extrapola o uso dos conhecimentos e tecnologias médico-sanitárias $e$ inclui tecnologias de comunicação social que estimulam a mobilização, organização e atuação dos diversos grupos na promoção e na defesa das condições de vida e saúde.

As formas de organização dos processos de trabalho envolvidas em cada um desses modelos são diversas. Do trabalbo intensivo condensado na rede de prestação de serviços de sanide, cujo locus privilegiado no modelo médicoassistencial é o hospital, passa-se, no modelo sanitarista, para as unidades de saúde, a partir das quais se operacionalizam as campanhas, programas e ações de vigilancia epidemiológica e sanitária. A proposta de Vigilância da Saúde, entretanto, transcende os espaços institucionalizados do "sistema de serviços de saúde" $e$ se expande a outros setores e órgãos de ação governamental e não governamental, envolvendo uma trama complexa de entidades representativas dos interesses de diversos grupos sociais.
Em sintese, a Vigilancia da Saúde apresenta sete caracteristicas básicas: a) Intervenção sobre problemas de saúde, (danos, riscos e/ ou determinantes); b) Ênfase em problemas que requerem atenção $e$ acompanhamento contínuos; c) Operacionalização do conceito de risco; d) Articulação entre ações promocionais, preventivas $e$ curativas; e) Atuação intersetorial; $f$ ) Açôes sobre o território; g) Intervenção sob a forma de operações.

A Vigilância da Saúde corresponderia, assim, a um modelo assistencial que incorpora e supera os modelos vigentes, implicando a redefinição do objeto, dos meios de trabalho, das atividades, das relações técnicas e sociais, bem como das organizaçôes de saúde e da cultura sanitária. Nessa perspectiva, aponta na direção da superação da dicotomia entre as chamadas práticas coletivas (vigilância epidemiológica e sanitária) e as práticas individuais (assistência ambulatorial e hospitalar) através da incorporação das contribuiçóes da nova geografia, do planejamento urbano, da epidemiologia, da administração estratégica e das ciências sociais em saúde, tendo como suporte politico-institucional o processo de descentralização e de reorganização dos serviços $e$ das práticas de sauide ao nivel local. 
A operacionalização da vigilância da saúde no município

Várias propostas e recomendações especificas visando à criação de condiçôes para a operacionalização da vigilância da sande têm sido feitas em vários encontros, seminários e congressos realizados nos últimos anos, valendo a pena ressaltar duas questões centrais: a capacitação de pessoal para o exercício das atividades de vigilancia, na perspectiva ampliada que foi sendo construida, e o aperfeiçoamento dos sistemas de informação visando à expansão das bases de dados, à qualidade das informações $e$ à articulação entre os diversos subsistemas, inclusive os derivados da atenção médicohospitalar.

A primeira questão foi objeto de uma proposta elaborada sob patrocínio do CENEPI, tratando de superar o modelo pedagógico dos cursos de epidemiologia realizados no inicio dos anos 90 e incorporando a metodologia de planejamento estratégico situacional, para a formação e capacitação em vigilância da saúde. ${ }^{38}$

A segunda questão foi objeto das duas outras Oficinas de Trabalho realizadas em Congressos de Epidemiologia, nas quais foram sistematizadas várias recomendações: a) elaboração de propostas de padronização e compatibilização dos principais bancos de dados nacionais; b) elaboração de estratégia para a capacitação em larga escala de recursos humanos em nivel municipal, estadual e federal, para a utilização dos bancos de dados existentes preparando-os para análise, planejamento $e$ avaliação de saúde, com a finalidade de utilizar a informação para a tomada de decisões; c) construção de uma Base Minima de Dados Municipais (BMIM), integrando na mesma unidade geográfica informações demográficas, socioeconômicas e epidemiológicas necessárias para o gerenciamento do SUS. ${ }^{39,40}$

No momento atual, a elaboração de propostas de operacionalização da vigilância toma como eixo central o processo de municipalização. Considerando os incentivos financeiros previstos na NOB 96, as ações de capacitação de pessoal e cooperação técnica previstas no VIGISUS, a possibilidade de assessoria por parte das SES e instituições acadêmicas, o municipio é posto diante do desafio de reorientar o conjunto de ações e serviços desenvolvidos no sistema municipal de sauide, quais sejam: a) Assumir e consolidar a Vigiláncia epidemiológica; b) Assumir e consolidar a Vigilância sanitária; c) Assumir e implementar os programas de sauide da familia; d) Reorganizar o perfil de oferta das unidades básicas, considerando os programas especiais e o perfil epidemiológico da população; e) Articular a atenção de média e alta complexidade, fortalecendo a rede pública e renegociando a compra de serviços ao setor privado; f) Redefinir a assistência laboratorial e farmacêutica.

Levando em conta a heterogeneidade das situações dos municípios, mais do que implementar as propostas e diretrizes emanadas do nivel federal e estadual, o desafio maior para os prefeitos e secretários municipais de saúde é definir a linba com que pão conduzir a politica de saúde municipal articulando distintos elementos gerenciais, financeiros, programáticos, organizativos e operacionais.

Adotar a concepção ampliada de Vigilância da Saúde, visando à transformação do modelo de atenção à saúde ao nivel municipal, implica, em primeiro lugar, avançar no processo de municipalização da gestão do sistema e da gerência das unidades de saúde localizadas no território dos municípios. Em segundo lugar, implica investir na articulação intersetorial, na reorganização da atenção primária (oferta organizada e ações de promoção da saúde e prevenção de riscos e agravos, partindo dos territórios da "saúde da familia", aos territórios distrital e municipal) e no fortalecimento do controle social sobre a gestão do sistema de saúde.

Trata-se, portanto, de desencadear um processo de construção das práticas de Vigilância da Saúde, tendo como eixo central o trinômio 
"informação-decisão-ação",, ${ }^{41}$ que se traduz do ponto de vista técnico-operacional, no uso de métodos/técnicas de planejamento que dêem suporte ao processo de identificação e priorização de problemas de grupos populacionais de territórios delimitados e à articulação de operações integradas de promoção, prevenção, recuperaçãa $e$ reabilitação destinadas ao enfrentamento contínuo dos problemas selecionados.

$O$ ponto de partida para o desencadeamento do processo de planejamento da vigilância à saúde é a Territorialização do sistema municipal de saúde, isto é, o reconbecimento e o esquadrinbamento do território do município segundo a lógica das relações entre condições de vida, saúde e acesso às ações e serviços de saúde. Isto implica um processo de coleta e sistematização de dados demográficos, socioeconômicos, politicoculturais, epidemiológicos e sanitários que, posteriormente, devem ser sistematizados de modo a se construirem o mapa básico e os mapas temáticos do municipio. ${ }^{22,43,44,45}$

Um mapa básico contém a delimitação territorial do municipio, com o desenho da configuração urbano-rural, ou seja, a delimitação dos distritos, bairros, ruas, contemplando o adensamento demográfico da população. Os mapas temáticos implicam, em primeiro lugar, localização espacial dos seviços de sauide e outros equipamentos sociais, como creches, escolas, igrejas, etc., com a delimitação das vias de acesso da população aos serviços, o que já dá uma idéia dos fluxos de demanda às diversas unidades de saúde do municipio. Em segundo lugar, deve-se fazer a caracterização dos diversos grupos populacionais do municipio segundo suas condições de vida, o que permitirá a justaposiçãa do mapa básico com o mapa temático dos serviços de saúde e o mapa temático das condiçôes de vida. Finalmente, é necessário fazer a distribuição espacial dos principais problemas de saude, identificados em função de informações epidemiológicas extraidas de bancos de dados oficiais ou obtidas através de "estimativa rápida" com "informanteschave", cruzando estas informações com os mapas elaborados anteriormente. ${ }^{45}$

$O$ processamento das informações $e$ a sua projeção em mapas permite a identificação de vários territórios superpostos no ámbito do município. Assim é que as experiências de distritalização em curso permitiram que se avançasse para a identificação do "território distrito" (ou municipio, caso este corresponda a um DS), cuja base é geográfico-populacional, configurada segundo a distribuição da população nos vários aglomerados urbanos (bairros, favelas, invasões, etc.), ao qual se superpõe o "território área de abrangência das unidades de saúde", delimitadas em função da demanda aos serviços. Em seguida, é possivel delimitar as "microáreas" em função das condições de vida e mapear os principais problemas que atingem grupos populacionais $e$ até grupos de famílias especificos em determinadas ruas e bairros (Figura 5).

O propósito fundamental desse processo de territorialização é permitir a definição de prioridades em termos de problemas e grupos, o mais aproximadamente possivel, o que se refletirá na definição das açôes mais adequadas, de acordo com a natureza dos problemas identificados, bem como na concentração de intervenções sobre grupos priorizados $e$, conseqüentemente, em um maior impacto positivo sobre os niveis de sauide e as condiçôes de vida. Trata-se do uso inteligente da epidemiologia, através da "microlocalização dos problemas de saúde, a intervenção no ámbito populacional pantada no saber epidemiológico $e$ a apropriação de informações acerca do território-processo, visando à integralidade, à intersetorialidade, à efetividade e à eqüidade". ${ }^{3}$

Uma vez que se conte com a territorialização do município em função das condições de vida e saúde, enquanto parte da análise da situação de saúde, é possivel dar seguimento ao processo de planejamento $e$ programação local que não se esgota na mera racionalização da oferta de serviços 
Figura 5 - Territorialização dos Problemas e das Ações de Saúde no Município

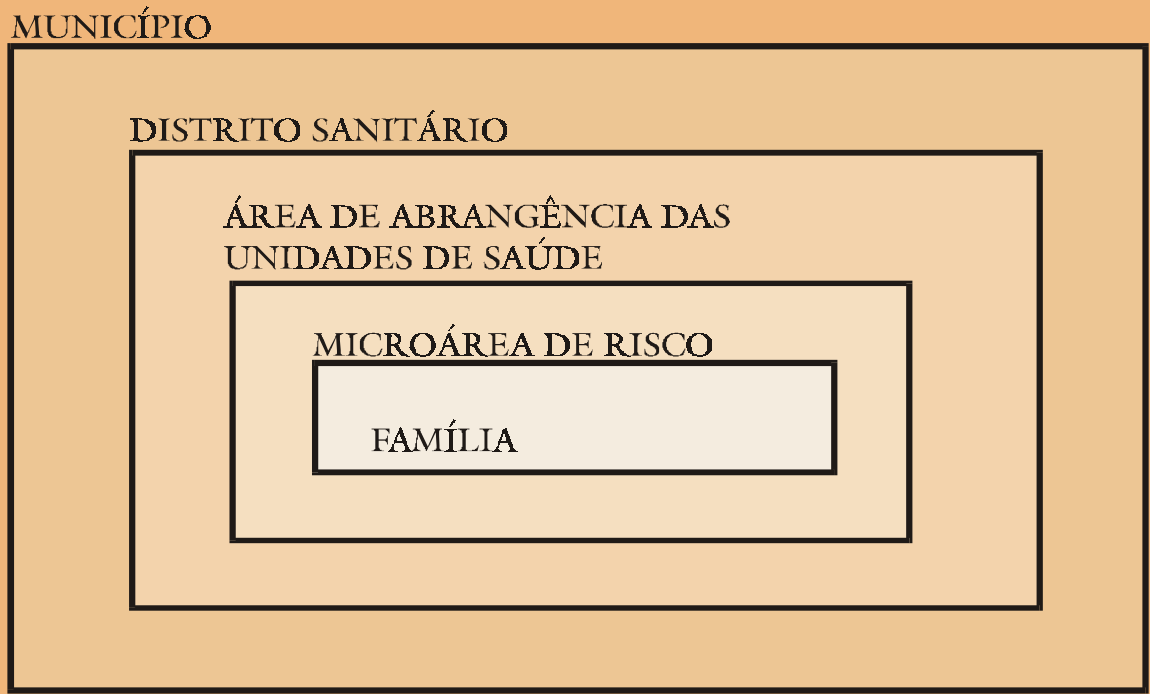

ambulatoriais e hospitalares, tal como ocorren na maioria dos estados durante o primeiro movimento da Programação Pactuada Integrada - PPI. Sem negar a importáncia da utilização do planejamento como instrumento de racionalização, a construção da Vigilância da Saude exige a utilização de um enfoque mais abrangente, situacional, que incorpore como objeto de intervenção os problemas de saúde e seus determinantes. ${ }^{46,47}$

Nessa perspectiva, o planejamento e a programação não se resumem a uma simples técnica que pode ser reproduzida em qualquer circunstância de tempo ou lugar, senão que envolve sujeitos dotados de vontade politica, o que significa dizer que podem ser não apenas instrumentos de manutenção de uma determinada situação, mas também podem ser instrumentos de mudança e de transformação desta situação. ${ }^{48}$ Não se trata de fazer tudo que é possivel tecnicamente, e sim aquilo que é necessário para dar conta dos Problemas reais existentes na população de um determinado território, seja este uma microárea onde se localizam familias em condiçôes de vida precárias, em uma área de abrangência de uma Unidade de Saúde ou no municipio como um todo.

Planejar a partir da identificação, descrição e análise dos determinantes sociais dos problemas de saúde implica a definição dos objetivos, metas, ações e atividades que serão realizadas para o enfrentamento dos problemas de saude identificados e priorizados na área, com o estabelecimento dos responsáveis, prazos e recursos envolvidos. Do ponto de vista metodológico, o planejamento e programação situacional em saude tem-se fundamentado na chamada "trilogia matusiana" - o PES, o MAPP e o ZOOP, ${ }^{49,50}$ originando propostas de Planejamento e programação local - PPLS orientadas para a operacionalização da Vigilância da Saúde.

O que importa ressaltar é que, com a utilização desse enfoque, o municipio pode construir uma "árvore de problemas" ou um "fluxograma situacional" para sistematizar as 
informações acerca dos problemas de saude, subsidiando assim um processo de tomada de decisões com relação ao "que fazer" para enfrentá-los. Essa decisões contemplam uma "árvore de objetivos" dos quais derivam as ações a serem realizadas nos territórios considerados em uma perspectiva intersetorial. Ou seja, as ações e serviços a serem desenvolvidos não se restringem àqueles que já são tradicionalmente ofertados pelas unidades de saúde, envolvendo um esforço adicional de mobilização e articulação de outros órgãos governamentais e não governamentais que atuam na área, inclusive a mobilização e envolvimento dos individuos, das familias e das coletividades que vivem e trabalham neste local.

$O$ conjunto das ações e serviços definidos para o enfrentamento dos problemas selecionados constituem as Operações a serem implementadas, segundo uma lógica que privilegie, não a organização de estruturas burocráticas para administrar os recursos humanos e materiais envolvidos e sim na perspectiva de flexibilização gerencial (por projetos) que implica a reorganização de equipes de trabalho e gerenciamento descentralizado e modular, ${ }^{51,52}$ privilegiando-se o controle gerencial e social do processo de implementação das ações.

Do ponto de vista do seu conteúdo, as Operações definidas no ámbito do municipio poderão incorporar desde ações politicas, de mobilização social no ámbito de organizações governamentais e não governamentais, até ações de saude propriamente ditas, envolvendo a educação sanitária e comunicação social dirigidas a grupos específicos em função da distribuição social dos problemas de saúde, a ações de vigiláncia epidemiológica, sanitária, nutricional, até serviços de assistência direta a pessoas, ao nivel ambulatorial e hospitalar. Cabe ressaltar a necessidade de adequação das ações propostas aos determinantes e condicionantes dos problemas, bem como às suas expressões fenomênicas (riscos e danos).

$O$ desencadeamento de um processo de fortalecimento da autonomia politico-gerencial dos municípios e da elevação da sua capacidade técnico-operacional de planejamento, programação, controle gerencial e operacionalização de ações voltadas ao enfrentamento dos problemas de saúde em seu território faz parte, sem dúvida, do processo de reconstrução do Estado no momento atual. Para os municipios significa, concretamente, a possibilidade de, a partir das iniciativas em Saúde, reestruturarem a gestão Municipal em seu conjunto, em uma perspectiva democrática, participativa, tecnicamente competente $e$ gerencialmente eficiente e efetiva.

$\mathrm{Na}$ perspectiva técnica da implementação da vigilância da saúde, a metodologia do planejamento e programação poderia ser aplicada em distintos momentos com a seguinte seqüência lógica:

a) análise da situação de saúde;

b) desenho de situação-objetivo;

c) desenho das estratégias;

d) programação, acompanbamento e avaliação.

Para os municípios pequenos e médios que decidam apostar na construção dessa proposta, alguns passos podem ser sugeridos para sua implantação e operacionalização:

a) sensibilizar técnicos para desencadear a construção da proposta no ámbito institucional;

b) compor uma equipe minima (dois ou três técnicos) capaz de reunir material bibliográfico e técnico sobre vigiláncia da saúde $e$ de se articular com especialistas da Secretaria Estadual, de universidades e do Ministério da Saúde;

c) assumir esta equipe minima enquanto um "grupo organizado para a ação" em vez de investir, inicialmente, na organização de uma estrutura ou órgão (departamento, divisão, setor, serviço, etc.);

d) estimular o entrosamento deste grupo com técnicos responsáveis por programas $e$ serviços afins aos modelo de vigiláncia da saúde no município, a exemplo do PACS/PSF, vigilância epidemiológica, vigiláncia sanitária, 
SUS, Modelos Assistenciais e Vigilância da Saúde

saúde ambiental, saude ocupacional, programas especiais de controle de doenças e agravos, etc.;

e) garantir a capacitação e educação permanente da equipe inicial e programar o desenvolvimento de recursos humanos para a expansão do modelo;

f) reunir e sistematizar as informações disponiveis para identificar e priorizar os problemas de saude que justificam um acompanhamento e atenção continuos (ver planos municipais de saúde, anuários estatísticos de base municipal. Bases de dados disponibilizados pelo MS, etc.);

g) planejar e programar as operações (setoriais e intersetoriais) para enfrentamento continuado dos problemas selecionados, incluindo a montagem do sistema de informações em saúde;

b) montar uma gerência de operações e de projetos para execução, acompanbamento $e$ avaliação dos mesmos.

\section{Comentários finais}

A opção por determinado modelo de atenção não está isenta de finalidades e valores, explicitas ou implícitos. Um mesmo rótulo ou proposta pode expressar-se, concretamente, em práticas distintas. De um modo ou de outro, tal proposta será aquilo que, em cada situação concreta, os sujeitos sociais, submetidos a determinadas relaçôes econômicas, politicas e ideológicas, conseguirem imprimir da marca dos seus projetos.

O essencial, nos parece, todavia, é que a adoção da perspectiva da Vigilância da Saúde, enquanto eixo da reorientação do modelo assistencial do SUS, aponta caminhos para a superação da crise do sistema de saúde que levam em conta a realidade de cada município, tanto do ponto de vista politico e cultural, quanto do ponto de vista social, epidemiológico e sanitário.

Planejar e programar o desenvolvimento da Vigilancia da Saúde em um território especifico exige um conbecimento detalhado das condiçôes de vida e trabalho das pessoas que ai residem, bem como das formas de organização $e$ de atuação dos diversos órgãos governamentais e não governamentais, para que se possa ter "visão estratégica”, isto é, clareza sobre o que é necessário e possivel de ser feito.

Exige também uma disponibilidade e um interesse muito grande em se envolver um uma ação comunicativa, isto é, em participar de um diálogo permanente com os representantes destes órgãos, com os representantes dos grupos sociais existentes e com as pessoas, de um modo geral, buscando envolvêlas em um trabalho coletivo cujo propósito maior é a reestruturação da ação coletiva em defesa da Saúde e da melhorias das condiçoes de vida. Os saberes de planificação e a tecnologia de gestão disponiveis representam ferramentas significativas para a construção coletiva dessa proposta. $^{53}$

Finalmente, cabe considerar certos limites da reorganização das práticas sanitárias na perspectiva da vigilância da saúde. $O$ primeiro ponto a destacar é a preocupação com a questão da mudança das condições de saúde, de melhorar o nivel de saúde da população. A preocupação com o impacto nas experiências de implantação do modelo assistencial da vigilância da saúde não significa apenas um viés sanitarista. E, particularmente, politico. $O$ segundo ponto a ressaltar é a necessidade de contextualização, de modo que o modelo seja entendido como racionalidade e não como receita, prescrição normativa. Examinar, concretamente, o processo politico em que está inserido. Trata-se de superar as dificuldades em trazer a questão do poder ao se discutir vigilância da saúde.

Temos a obrigação de contextualizar nosso discurso, de ver o que está acontecendo com a realidade na qual nos inserimos. Estamos vivendo processos politicos muito importantes ao nivel nacional, inclusive na área de vigiláncia. $A$ direção do Centro Nacional de Epidemiologia tem mudado diversas vezes. Que compromissos este centro tinha com a proposta de vigilancia 
da saude? Quais os que pretende ter agora? Quais foram os motivos que levaram a essas mudanças? Seriam técnicos? Que repercussões podem ser esperadas para a epidemiologia e para o campo da Savide Coletiva? Será que a análise da situação de saúde fundamenta a prioridade que parece se conceder a certos problemas de sauide?

Assim, é pertinente considerar a questão do poder para analisar a viabilidade da implantaçãa e expansão da vigilância da saúde nos estados e, especialmente, nos municipios. A reorganização das práticas de saúde representa uma possibilidade teórica e um dos possiveis históricos da reorientação de sistemas de saúde. Insere-se na "região" dos modelos assistenciais, entendidos como combinação de tecnologias acionadas para o enfrentamento de problemas (danos e riscos) e necessidades de saude (incluidas as carências e problemas, mas, também, oportunidades e projetos de vida pessoal ou de classe dos sujeitos sociais). Seguramente, outros projetos estão em curso, competindo ou até mesmo dominando a vigilância da saúde que formulamos, implementamos, acreditamos ou apostamos.

Esta reorganização das práticas de saúde pode envolver a recomposição dos meios de trabalho, a reestruturação das atividades (trabalho propriamente dito) dos agentes, $e$ a redefinição das relações sociais e técnicas sob as quais se realiza o processo de trabalho. Cada uma dessas modificações pode ser estimulada pela identificação de novos problemas ou necessidades de saúde, bem como elaboração de conceituações distintas acerca do objeto dessas práticas. "Habitus" ou culturas organizacionais podem também potencializar ou neutralizar esses movimentos de reorganização das práticas.

A operacionalização dessas idéias supõe identificar novas maneiras de pensar o processo de trabalho em saude. Portanto, independentemente das diversas concepções acerca da vigilância, é possivel destacar a preocupação com o impacto sobre o estado de saúde da população $e$ a situação epidemiológica, isto é, com o impacto sobre os danos, riscos e os determinantes das necessidades sociais de saúde. Isto significa a possibilidade de reconceituação do objeto das práticas de saude e, por conseguinte, a formulação de indagações sobre a pertinência, consistência ou eficácia dos meios de trabalbo $e$ do trabalho propriamente dito utilizados para a apreensão e/ou transformação desse objeto.

$O$ processo de distritalização (especialmente quando os distritos sanitários valorizam a dimensão técnica dos modelos assistenciais) e as açôes programáticas de saúde, no ámbito local, podem alimentar, ao nivel dos serviços de sauide, os movimentos de redefinição de práticas, inclusive na perspectiva da vigilância da saúde. Na medida em que se processa, no ámbito dos serviços, a reconceitualização do objeto das práticas de sanide e, quando for o caso, a reorganização do processo de trabalho dos agentes e a readequação dos instrumentos do trabalho (sejam tecnologias materiais ou não materiais), importa indagar se tais fatos, ao serem produzidos, geraram acumulações que impactaram a situação de sauide e em que tempos político e técnico.

Por que insistir nessas questões? Porque essas idéias que estamos trazendo, desenvolvendo e debatendo - que não são, necessariamente, novas, têm a ver com a possibilidade de que elas se construam e se constituam em novas realidades pela práxis dos sujeitos sociais. A preocupação do quanto se possa ou se deva gerar poder para poder continuar atuando é fundamental. Se não pretendemos que essas experiências permaneçam como "estudo piloto" precisamos, urgentemente, torná-las hegemônicas no sentido gramsciano. Sermos capazes de imprimir uma direção cultural e uma direcionalidade prática no cotidiano para o nosso projeto. Mesmo sem ser voluntaristas, temos que estar atentos para os movimentos do xadrez politico no sentido de construir viabilidade para tal projeto na 
perspectiva da Reforma Sanitária como resposta criativa à contra-reforma que nos estão fazendo engolir como se fora a única saida.

Não adianta muito insistirmos nas lembranças dos espaços de trabalho conquistados nos governos que possibilitaram essas inovações conceituais e técnicas. Os que chegaram depois todos conhecemos, especialmente o pavor deles diante do novo. Estas são questões muito concretas que nós precisamos debater. Perguntarmo-nos sempre em que medida essas práticas inovadoras que estamos construindo conseguem, efetivamente, criar poder para poder fazer melhor as coisas.

\section{Bibliografia}

1. Klekowsky BM, Roemer M, Werff AVD. Sistemas Nacionales de Salud y su Reorientación hacia la Salud para Todos. Pautas para una politica. Cuardernos de Salud Pública 77:134, 1984.

2. BRASIL, Ministério da Administração Federal e Reforma do Estado. Plano Diretor da Reforma do Aparelho de Estado. Brasilia, Presidência da República, Imprensa Nacional, 1995.

3. Mendes EV. Distrito Sanitário: o processo social de mudança das práticas sanitárias do Sistema Único de Saude. HUCITECABRASCO, São Paulo - Rio de Janeiro, 1993.

4. Paim JS. A Reforma Sanitária e os Modelos Assistenciais. In: Rouquayrol $M Z$, Epidemiologia \& Saúde, $4^{a} \mathrm{ed}$, MEDSI, Rio de Janeiro, p.455 - 466, 1994.

5. Paim JS. A reorganização das práticas de saude em Distritos Sanitários In: Mendes EV, Distrito Sanitário: o processo social de mudanca das práticas sanitárias do Sistema Unico de Saude, HUCITECABRASCO, São Paulo - Rio de Janeiro, $p$. 187 - 220, 1993.

6. Teixeira CF, Melo C. (orgs.). Construindo Distritos Sanitários: a experiência da cooperação Italiana em Saúde no município de São Paulo, HUVITEC/CIS, São Paulo - Salvador, 1995.

7. Marinho de Souza MF, Kalighman AO. Vigilância à Saúde: Epidemiologia, Serviços e Qualidade de Vida. In: Rouquayrol, $M Z$, Epidemiologia \& Saúde, $4^{a}$ ed., MEDSI, Rio de Janeiro, $p$. 467-476, 1994.

8. BRASIL, Ministério da Saúde. $10^{a}$ Conferência Nacional de Saúde, Anais. Brasilia, DF, 1996.

9. Carta de Fortaleza. Saúde em Debate 42:77-78, 1995.

10. Paim JS. Organização em serviços de saude: modelos assistenciais e práticas de saúde. Salvador, p.25, 1996. (mimeo)

11. BRASIL, Ministério da Saúde. Norma Operacional Básica 001/93, 1993.

12. Paim JS. Medicina familiar no Brasil: movimento ideológico e ação politica. In: Saude, crises e reformas, UFBA-PROED, Salvador, Bahia, p. 151-183, 1986.

13. BRASIL, Ministério da Saúde, CENEPI. VIGISUS, 1998.

14. BRASIL, Ministério da Saúde. Norma Operacional Básica 001/96, 1996.

15. Mendes EV. O consenso do discurso e o dissenso da prática social: notas sobre a municipalização da saúde no Brasil. São Paulo, s. n. t., p.11, 1991.

16. Mendes EV. Uma agenda para a saúde. $1^{a}$. ed. HUCITEC, São Paulo, 1996.

17. Garcia JC. A articulação da medicina e da educação na estrutura social. In: Nunes E (org.), Juan César Garcia: pensamento social em saúde na América Latina, Cortez/ ABRASCO, São Paulo, p.189-232, 1989.

18. Silva GR. Avaliação e perspectivas da Epidemiologia no Brasil. $1^{0}$ Congresso Brasileiro de Epidemiologia, Anais. UNICAMP/ABRASCO, Campinas, SP, p.183-187, 1990. 
19. Barata R. Reorientação das práticas de vigiláncia epidemiológica. In: Seminário Nacional de Vigilância Epidemiológica. Anais. Ministério da Saúde, Fundação Nacional de Saúde-CENEPI, Brasília, p. 63$68,1992$.

20. Almeida Filho N, Rouquayrol $M Z$. Introdução à Epidemiologia Moderna. Apce/ ABRASCO, Salvador, p.222, 1990.

21. Barreto $M L$, Carmo EH. Situação de sauide da população brasileira: tendências históricas, determinantes e implicações para as politicas de saúde. Informe Epidemiológico do SUS 3/4:7-34, 1994.

22. Paim JS, Teixeira MG. Reorganização do sistema de vigiláncia epidemiológica na perspectiva do Sistema Único de Saúde (SUS). Seminário Nacional de Vigilancia Epidemiológica, Anais. Ministério da Saúde, Fundação Nacional de SaúdeCENEPI, p.93-144, 1992.

23. Costa E. A Vigilancia Sanitária: defesa e proteção da saúde. Tese de Doutorado. Faculdade de Saúde Pública, Universidade de São Paulo, São Paulo, 1998.

24. BRASIL, Lei $n^{\circ}$ 6.259. Brasilia, 1975.

25. I Congresso Brasileiro de Epidemiologia. Vigilância Epidemiológica - Reformulação do sistema face à municipalização dos serviços de saude. Realtório de Oficina de Trabalho. Anais. UNICAMP-ABRASCO. Campinas, SP, p.33-36, 1990.

26. III Congresso Brasileiro de Epidemiologia. Vigilância à Saúde. Relatório de Oficina de Trabalho. Salvador, Babia, p.11, 1997.

27. Scharaiber LB. Epidemiologia em serviços: uma tecnologia de que tipo? Informe Epidemiológico do SUS 3:532, 1995.

28. Scharaiber LB. Politicas públicas e planejamento nas práticas de saúde. Saúde em Debate 47:28-35, 1995.
29. OPS. Usos e perspectivas da Epidemiologia, Documentos del Seminario. Publicación PNSP 84-47, Washington, D. C. p.243, 1984.

30. St John R. La necessidad de un pensamiento epidemiológico en los servicios de salud y la formación de recursos bumanos. In: OPS. La formación en Epidemiologia para el desarrollo de los servicios de salud. Série Desarrollo de Recursos Humanos, $n^{\circ} 88$, Washington, D.C., p.19-24, 1987.

31. Castellanos PL. Epidemiologia y organización de los servicios. In: OPS/ OMS. La formación en epidemiologia para el desarrollo de los servicios de salud. Série Desarrolllo de Recursos Humanos, $n^{\circ} 88$, Washington, D.C., p.30-40, 1987.

32. OPS. Promoción de liderazgo y formación avanzada en Salud Pública: la prestación de servicios de salud. Educación Médica y Salud 3:193-425, 1992.

33. Silva GR. A epidemiologia na organização dos serviços de saúde. In: Costa MF, Souza RP (orgs.). Qualidade de Vida: compromisso histórico da Epidemiologia. COOPMED/ABRASCO, Belo Horizonte, p.108-139, 1994.

34. Mendonça EF, Cosenza GW, Carvalho DM, Gutierrez EB, Sevalho G, Ribeiro JGL, Toledo L, Alfradique MEM, Teixeira MG, Carvalho MS, Liebel M, Oliveira OL, Ladeira RM. Repensando a vigiláncia epidemiológica. Relatório de Oficina de Trabalho. II Congresso Brasileiro de Epidemiologia. In: Costa MF, Souza RP (orgs.) Qualidade de Vida: compromisso histórico da Epidemiologia, COOPMEED/ABRASCO, Belo Horizonte, p.277-280, 1994.

35. I Congresso Brasileiro de Epidemiologia, Anais. Análise de programas de controle de doenças - avaliação de suas estratégias de integração ao SUS. Relatóro de Oficina de 
SUS, Modelos Assistenciais e Vigilância da Saúde

Trabalho. UNICAMP/ABRASCO, Campinas, SP, p. 37-43, 1990.

36. Waldman CA. As concepções de vigilância como instrumento de saúde pública e a implantação do SUS. In: Seminário Nacional de Vigilancia Epidemiológica, Anais. Ministério da Saúde, Fundação Nacional de Saúde-CENEPI, Brasilia, p.45-51, 1992.

37. Teixeira CF. Epidemiologia e Planejamento em Saúde: contribuição ao estudo da prática epidemiológica no Brasil 19901995. Tese de Doutorado. Instituto de Saúde Coletiva, Universidade Federal da Babia, Salvador, Babia, 1996.

38. Teixeira CF, Pinto L. A formação de pessoal em Vigilancia da Saúde. Informe Epidemiológico do SUS 6:5-21, 1993.

39. Morais I. Utilização de grandes bancos de dados nacionais. Relatório de Oficina de Trabalho. II Congresso Brasileiro de Epidemiologia. In: Costa MF, Souza RP (orgs.), Qualidade de Vida: compromisso histórico da Epidemiologia, COOPMEED/ABRASCO, Belo Horizonte, MG, p. 285-290, 1994.

40. Morais I. Informações em Saúde: da prática fragmentada ao exercicio da cidadania. HUCITEC/ABRASCO, São Paulo, p.172, $1994 b$.

41. Fosaert H, Llopis A, Tigre CH. Sistemas de Vigilância Epidemiológica. Boletim Oficina Sanitaria Panamericana 76:512-525, 1974.

42. Unglert C. Territorialização em sistemas de saude. In: Mendes EV. Distrito Sanitário: o processo social de mudança das práticas sanitárias do Sistema Único de Saúde, HUCITEC-ABRASCO, São Paulo - Rio de Janeiro, p.221-235, 1993.

43. Kadt E, Tasca R. Promovendo a equidade: um novo enfoque com base no setor da
Saúde. HUCITEC/Cooperação Italiana em Saúde, São Paulo - Salvador, p.107, 1993.

44. Tasca R. Sistemas de informação em saúde para Distritos Sanitários In: Mendes EV. Distrito Sanitário: o processo social de mudança das práticas sanitárias do Sistema Único de Saúde, HUCITEC-ABRASCO, São Paulo - Rio de Janeiro, 1993.

45. Notarbartolo di Villarosa F. A estimativa rápida e a divisão do território no distrito sanitário. Manual de instruções. OPS, Série Desenvolvimento de Serviços de Sauide, $n^{0}$ 11, p.54, 1993.

46. Teixeira CF. Planejamento e programação situacional em distritos sanitários. In: Mendes EV. Distrito Sanitário: o processo social de mudança das práticas sanitárias do Sistema Único de Saúde. HUCITECABRASCO, São Paulo - Rio de Janeiro, p.237 - 265, 1993.

47. Teixeira CF. Planejamento e programação da Vigilancia da Saúde. In: ISC/UFBA, Politica de Saúde: Coletânea de textos para a Disciplina ISC-003. Salvador, p.16, 1997 (mimeo).

48. Testa M. Pensar em Sauide. Intermédica, Porto Alegre, Rio Grande do Sul, 1991.

49. Artmann E. O planejamento estratégicosituacional: a trilogia matusiana e uma proposta para o nivel local de saúde (uma abordagem comunicativa). Tese de Mestrado, ENSP/FIOCRUZ, Rio de Janeiro, 1993.

50. Mendes E. Planejamento e programação local da Vigilancia da Saúde no Distrito Sanitário. OPS, Série Desenvolvimento de Serviços de Saúde, $n^{\circ}$ 13, 1994.

51. OPS/OMS. Desarrollo y fortalecimiento de los Sistemas Locales de Salud en la transformación de los Sistemas Nacionales de Salud: la administración estratégica. Washington, D.C., p.160, 1992.

52. Kliksberg B. Gerência social: dilemas 
gerenciais e experiências inovadoras In: Kliksberg B. (org.), Pobreza: uma questão inadiável, ENAP, Brasilia, p.127-146, 1994.

53. Merby EE. Em busca do tempo perdido: a micropolitica do trabalho vivo em saúde. In: Merhy EE, Onocko R. (org.), Agir em Saúde: um desafio para o público, HUCITEC/LUGAR EDITORIAL, São Paulo - Buenos Aires, p.385, 1997. 